\title{
The Modified Amplitude-Modulated Screening Technology for the High Printing Quality
}

\author{
Ivanna Dronjuk ${ }^{1}$, Maria Nazarkevych $\left.{ }^{2(}\right)$, and Oksana Troyan ${ }^{2}$ \\ 1 Automated Control Systems Department Institute of Computer Science, \\ National University Lviv Polytechnic, Lviv, Ukraine \\ idronjuk@polynet.lviv.ua \\ 2 Publishing Information Technology Department Institute of Computer Science, \\ National University Lviv Polytechnic, Lviv, Ukraine \\ mar.nazarkevych@gmail.com, troyan@gmail.com
}

\begin{abstract}
A new screening method based on the new form of screening element in improving printing quality was considered. The relationship between the Ateb-functions and the generalized superellipse is proved. Printing quality is an essential parameter when incorporating specially designed security features into the electronic file from which printing is done. Advisability of applying the proposed method for protection of information on the physical media was analyzed.
\end{abstract}

\section{Introduction}

The printing technology in computer epoch is completely changed. All details are described in classical books [1,2]. Digital screening is considered an algorithmic process that creates the images from an arrangement of small, binary dot elements. Generally in the different approaches for half-toning are two main screening methods: Amplitude Modulated and Frequency Modulated. Comparison of these two methods is described in [3]. Problem of improving printing quality using screening is concerned in [4]. The purpose of this study is to develop a modified amplitude-modulated screening method to improve the print quality. Improving the screening process can more accurately reflect the subtle elements of the image or text which makes protection of printed information on the physical media more reliable.

To implement the task, special protective graphics based on periodic Atebfunctions were built and the method of modified amplitude-modulated screening that allows the realization of printing fine detail and halftones with greater clarity was proposed.

This article continues the study, which was beginning in [5]. The modified amplitude-modulated screening technology allows to print small contours, lines and halftones with maximal precision.

\section{Mathematical Model}

Let us consider oscillation, as a nonlinear oscillating system with one degree of freedom. Modeling behavior of the system $x(t), y(t)$ is generated by a system of an ordinary differential equations in the form

(C) The Author(s) 2016

T. Czachórski et al. (Eds.): ISCIS 2016, CCIS 659, pp. 270-276, 2016.

DOI: $10.1007 / 978-3-319-47217-1 \_29$ 


$$
\left\{\begin{array}{l}
\frac{d x}{d t}+\beta y^{m}=0 \\
\frac{d y}{d t}+\alpha x^{n}=0
\end{array}\right.
$$

where $x(t), y(t)$ - are a values at time $t ; \alpha, \beta$ - constants that determine size of the oscillation period; $n, m$ - numbers that determine the degree of nonlinearity of the equation that affects the period of the main component of fluctuations. In the performance of such conditions on $\alpha, \beta$ and $n, m: \alpha \neq 0, \beta \neq 0, n=$ $\frac{2 k_{1}+1}{2 k_{2}+1}, k_{1}, k_{2}=0,1,2 \ldots, m=\frac{2 p_{1}+1}{2 p_{2}+1}, p_{1}, p_{2}=0,1,2 \ldots$ it is proved [6], that the analytical solution of equation (1) is represented as Ateb - functions.

The solution (1) is represented through periodic Ateb-functions [6] as follows

$$
\left\{\begin{array}{l}
x=C_{1} C a(n, m, \phi) \\
y=C_{2} S a(m, n, \phi) .
\end{array}\right.
$$

where $C_{1}, C_{2}$ are the some constants, $C a(n, m, \phi), S a(m, n, \phi)$ are Ateb-cosine and Ateb-sine respectively. Variable $\phi$ is associated with time $t$ as follows

$$
\phi=C_{3} t+\phi_{0}
$$

where $C_{3}$ - is some constant, $\phi_{0}$ - the initial phase of the oscillations, which are determined from the initial and periodical conditions for the system (1).

Periodical conditions are presented by expressions

$$
\left\{\begin{array}{l}
C a(n, m, \phi+2 \Pi)=C a(n, m, \phi), \\
S a(m, n, \phi+2 \Pi)=S a(m, n, \phi) .
\end{array}\right.
$$

where $\Pi$ is a half period of Ateb-function. Taking into account identity [2]

$$
C a(n, m, \phi)^{m+1}+S a(m, n, \phi)^{n+1}=1,
$$

we result following formula for a half period of Ateb-functions

$$
\Pi(m, n)=\frac{\Gamma\left(\frac{1}{n+1}\right) \Gamma\left(\frac{1}{m+1}\right)}{\Gamma\left(\frac{1}{n+1}+\frac{1}{m+1}\right)} .
$$

In formula (6) denomination $\Gamma(\bullet)$ means Gamma function. Identity (5) is a generalization of well-known trigonometrical identity $\cos ^{2} \phi+\sin ^{2} \phi=1$ in the case of Ateb-functions. So Ateb-functions generalize trigonometrical functions, if parameters $n=1$ and $m=1$, than $C a(1,1, \phi)=\cos \phi$ and $S a(1,1, \phi)=\sin \phi$.

\section{A Relationship Between the Ateb-Functions and the Generalized Superellipse}

In this section we show the relationship between the Ateb-functions and plane algebraic Lame's curves which is known also as a generalized superellipse. We propose to construct a unique raster element based on the Ateb-functions which we transform in a graphic element as a generalized superellipse. Representation 
superellipse by Ateb-functions enables a functional control under the screening element. Consider the generalized superellipse formula as follows [7]

$$
\left|\frac{x}{A}\right|^{p}+\left|\frac{y}{B}\right|^{q}=1 ; p, q>0
$$

where p, q, A and B are positive numbers. Let we substitute (2) into formula (7) we have a new formula

$$
\left|\frac{C_{1} C a(n, m, \phi)}{A}\right|^{p}+\left|\frac{C_{2} S a(m, n, \phi)}{B}\right|^{q}=1 .
$$

If we define $p=m+1, q=n+1$ and will select A and B that satisfy conditions $\frac{C_{1}}{A}=1, \frac{C_{2}}{B}=1$, we obtained exactly identity (5). Identity (8) shows a relationship between the Ateb-functions and the generalized superellipse. Thus we prove a new fact that main Ateb-function identity can be presented as a the generalized superellipse formula and periodical Ateb-cosine and Ateb-sine are strongly connected to the generalized superellipse.

We use formula (8) under conditions $n=m$ corresponding to the superellipse (not generalized) for constructing a new screening element. If we define $A_{1}=$ $\frac{A}{C_{1}}$ and $B_{1}=\frac{B}{C_{2}}$ we obtain the curve a new generalization of the generalized superellipse as

$$
\left|\frac{C a(n, m, \phi)}{A_{1}}\right|^{p}+\left|\frac{S a(m, n, \phi)}{B_{1}}\right|^{q}=1 .
$$

The further generalization of the superellipse is given in polar coordinates $(r, \phi)$ in case $r \neq 1$ by

$$
r(n, m, \phi)=\left|\frac{C a(n, m, \phi)}{A_{1}}\right|^{p}+\left|\frac{S a(m, n, \phi)}{B_{1}}\right|^{q} .
$$

We propose to name it the Ateb-superellipse. The area S inside the superellipse can be expressed in terms of the Gamma function as

$$
S=4^{1-\frac{1}{n+1}} \sqrt{\pi} A B \frac{\Gamma\left(1+\frac{1}{n+1}\right)}{\Gamma\left(\frac{1}{2}+\frac{1}{n+1}\right)},
$$

where $S$ defines the area of the proposed screening element.

\section{Technological Characteristics of the Screening Method}

A secure document must comply with International Standard ISO 14298:2013 specifies requirements for a security printing management system for security printers [8]. Safety elements should be made within 40-50 microns positive play and 60-80 microns reversed, and microprint size should be within 200250 microns, which guarantees high quality of printing and helps to reduce the likelihood of fraud. The authors have developed a new method for screening 


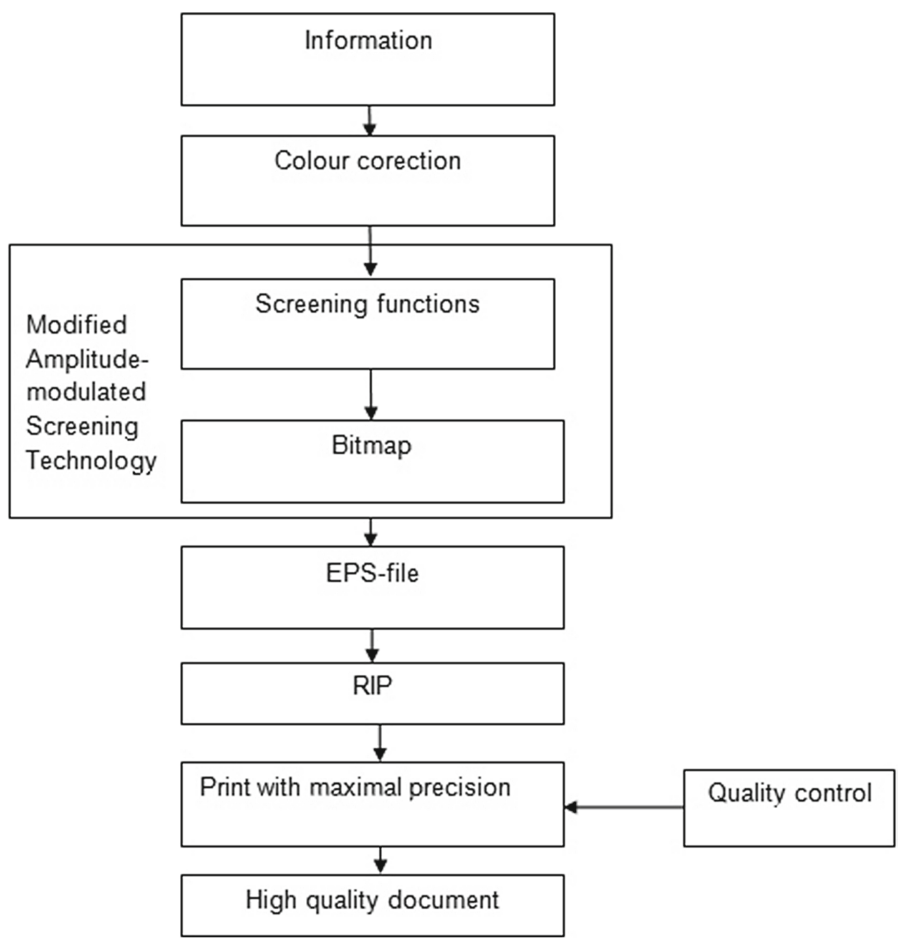

Fig. 1. Block diagram of screening technology

technology for improving printing quality. Figure 1 shows a block diagram of the proposed method. The resolution ability of print is restricted by the capacities of the output printing device.

It is important to provide high quality of the imprint for effective data protection on physical media. The better printed information is, the harder it is to forge it. Modern technologies allow faking everything, but there arises a question of economic criteria, namely the time and the cost of creating a fake. The main purpose of defense is to make the fake unprofitable. It is clear that the increase of print quality leads to higher cost of printed impression, and thus the cost of fraud rises. This is especially important for full-color prints, which are the most important documents (passport, driving license, etc.).

There is a problem of converting structure images in the process of printing, which is related to the difficulty of rendering fine detail and halftones. One of the most significant shortcomings of modern methods of structural transformation is much smaller resolution of the prints compared to the resolving ability of printing. This is due to the amplitude-modulated principle with binary halftone reproduction means of printing in which the tone values in a particular area of the original play with the relative area of the colored area of the print. Raster points are destroying contours and fine detail of halftone original, reducing the 
quality of the prints. Thus raster distortions are formed [9]. The magnitude and the visibility of raster distortion depend on screen frequency, frequency scanning function, and bitmap structure geometry and raster points. These raster distortions are associated with the parameters of amplitude-modulated screening such as pressure in the printing apparatus, ink supply, dot gain, sliding and double vision.

\section{Realization of the Screening Method}

A new screening method that can more accurately reproduce fine picture elements important for precision printing was developed. Improvement is achieved with the special structure of raster points which is better adapted to display halftones. Let consider a symmetric form of screening element, then $A=B=$ $A(i, j)$ and $n=m$ in a formula (8). The parameter $A(i, j)$ depends on the color intension of the screening points $(i, j)$. The formation of a screening point is the formula:

$$
T(i, j)=\left(\left|\frac{C a(n, n, \phi)}{A(i, j)}\right|^{n+1}+\left|\frac{S a(n, n, \phi)}{A(i, j)}\right|^{n+1}=1\right)_{(i, j)}
$$

where $i, j$ are the current coordinates of the screening points, $n$ is parameter of periodic Ateb-function. To send 8 bits of color depth raster point can take from 1 to 256 values, namely $j=1, \ldots, 16 ; i=1, \ldots, 16 ; 0 \leq \phi \leq 360$. Table 1 shows a unique screening elements for increasing colour intension. There is a comparison of a standard circle (row 1) and proposed screening elements (row 2). Table 2 presents calculation of the unique screening elements with parameter $n+1=e$ for colour intention from 5 to $100 \%$. For screening element we represent the colour intention as an area $S$ of screening element, where $S$ is calculating with formula (11). The point with a darker colour has a bigger screening element.

Development of the modified method of autotypical screening allows printing the fine details and halftones for text or graphical information on a physical medium more precisely which is shown in a Fig. 2. Figure 2 shows a large scale result of the screening method. The halftone reproduction is better for an image (b) than an image (a) for a normal size.

Table 1. The comparison of a standard circle and the unique elements of screening technology

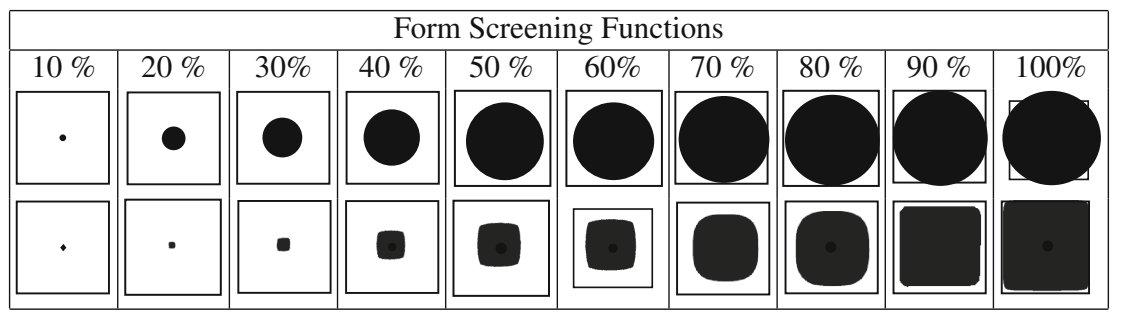


Table 2. Calculation table of the superellipse screening elements

\begin{tabular}{|c|c|c|c|c|}
\hline \multicolumn{5}{|l|}{ Superellipse } \\
\hline \multicolumn{3}{|l|}{ Basic parameters } & \multirow{2}{*}{$\begin{array}{l}\text { Perimeter } \\
\mathrm{P}, \mathrm{mkm}\end{array}$} & \multirow{2}{*}{$\begin{array}{l}\text { Area } \\
\mathrm{S}, \mathrm{mkm}^{2}\end{array}$} \\
\hline $\begin{array}{l}\text { A full size width } \\
(\mathrm{mkm}) \text { float }\end{array}$ & $\begin{array}{l}\text { B full size height } \\
(\mathrm{mkm}) \text { float }\end{array}$ & $\begin{array}{l}\mathrm{n}+1=\mathrm{e} \text { Ateb- } \\
\text { parameter, float }\end{array}$ & & \\
\hline 2.4 & 2.4 & 2.718 & 7.97 & 5.00 \\
\hline 3.5 & 3.5 & 2.718 & 11.62 & 10.01 \\
\hline 4.2 & 4.2 & 2.718 & 13.94 & 15.00 \\
\hline 4.9 & 4.9 & 2.718 & 16.27 & 20.10 \\
\hline 5.4 & 5.4 & 2.718 & 17.93 & 25.14 \\
\hline 5.9 & 5.9 & 2.718 & 19.59 & 30.00 \\
\hline 6.4 & 6.4 & 2.718 & 21.25 & 35.21 \\
\hline 6.8 & 6.8 & 2.718 & 22.58 & 40.00 \\
\hline 7.2 & 7.2 & 2.718 & 23.90 & 45.00 \\
\hline 7.6 & 7.6 & 2.718 & 25.23 & 50.02 \\
\hline 8 & 8 & 2.718 & 26.56 & 55.33 \\
\hline 8.4 & 8.4 & 2.718 & 27.89 & 60.00 \\
\hline 8.7 & 8.7 & 2.718 & 28.88 & 65.22 \\
\hline 9 & 9 & 2.718 & 29.88 & 70.00 \\
\hline 9.3 & 9.3 & 2.718 & 30.88 & 75.00 \\
\hline 9.6 & 9.6 & 2.718 & 31.87 & 80.11 \\
\hline 9.9 & 9.9 & 2.718 & 32.87 & 85.00 \\
\hline 10.3 & 10.3 & 2.718 & 34.20 & 90.59 \\
\hline 10.5 & 10.5 & 2.718 & 34.86 & 95.39 \\
\hline 10.8 & 10.8 & 2.718 & 35.86 & 100.00 \\
\hline
\end{tabular}

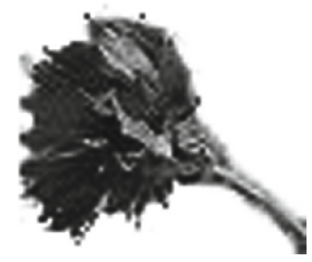

a)

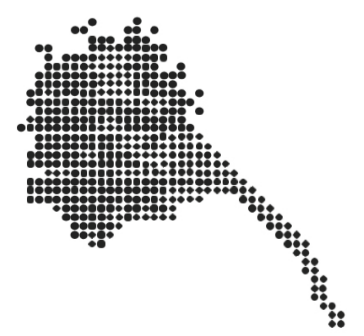

b)

Fig. 2. Comparision image with standard (a) and proposed (b) screening technology (scale 10:1) 


\section{Conclusion}

A new method of the forming a screening structure based on a periodic Atebfunctions is proposed. This structure is specially adapted for reproduction of fine protective graphical elements and halftones while printing, which improves the print quality greatly. The relationship between the Ateb-functions and the generalized superellipse is proved. Advantages of the method were shown in some experiment images. For improvement of this method we can construct asymmetric form of screening elements, and consider a screening point with an axis inclines at an angles $5^{\circ}-15^{\circ}$. This method can be used for improving the effectiveness of protecting information on paper, plastic and other material media.

Open Access. This chapter is distributed under the terms of the Creative Commons Attribution 4.0 International License (http://creativecommons.org/licenses/by/ $4.0 /$ ), which permits use, duplication, adaptation, distribution and reproduction in any medium or format, as long as you give appropriate credit to the original author(s) and the source, a link is provided to the Creative Commons license and any changes made are indicated.

The images or other third party material in this chapter are included in the work's Creative Commons license, unless indicated otherwise in the credit line; if such material is not included in the work's Creative Commons license and the respective action is not permitted by statutory regulation, users will need to obtain permission from the license holder to duplicate, adapt or reproduce the material.

\section{References}

1. Kipphan, H.: Handbook of Print Media. Springer, Heidelberg (2001). ISBN: 3-54067326-1

2. Bennett P., Romano, F., Levenson H.R.: The Handbook for Digital Printing and Variable - Data Printing, pp. 113-126. PIA/GATF Press, Pitsburgh, NPES (2007). ISBN: 978-5-98951-020-7

3. Sardjeva, R.: Investigation on halftoning methods in digital printing technology. Int. J. Graph. Multimed. (IJGM) 4(2), 1-10 (2013). ISSN: 09766448 (Print), ISSN: 09766456 (Online)

4. Sardjeva, R., Mollov, T.: Stochastic screening for improving printing quality in sheet fed offset. Int. J. Inf. Technol. Secur. 1, 63-74 (2012). ISSN: 1313-8251

5. Dronjuk, I., Nazarkevych, M., Medykovski, N., Gorodetska, O.: The method of information security based on micrographics. In: Kwiecień, A., Gaj, P., Stera, P. (eds.) CN 2012. Communications in Computer and Information Science, vol. 291, pp. 207-215. Springer, Heidelberg (2012)

6. Rosenberg, R.: The Ateb(h) functions and their proporties. Q. Appl. Math. 21(1), 37-47 (1963)

7. Sokolov, D.: Lame curve. In: Hazewinkel, M. (ed.) Encyclopedia of Mathematics. Springer, The Netherlands (2001). ISBN: 978-1-55608-010-4

8. ISO 14298: Management of security printing processes (2013).http://www.iso.org/ iso/home/store/catalogue_tc/catalogue_detail.htm?csnumber $=54594$

9. Kuznetsov, Y.V., Zheludev, D.E.: Method of objective evaluation the fine detail distortion in process of screening. In: IARIGAI, N 35, pp. 347-353 (2008) 